ESAIM: PROCEEDINGS AND SURVEYS, September 2014, Vol. 45, p. 209-218

J.-S. Dhersin, Editor

\title{
FLUID-STRUCTURE INTERACTION MODELING WITH CHIMERA GRIDS IN NSMB*
}

\author{
Yannick Hoarau, Thibaut Deloze, Anthony Ponce, Dorian Pena and Jan \\ DUŠEK ${ }^{1}$
}

\begin{abstract}
The paper presents the last few years of research at ICUBE, Team MECAFLU in the "Instabilité, Turbulence, Diphasique" group on the simulations of fluid-structure interactions applied to falling bodies using the NSMB solver. The first part of the study was devoted to the numerical study of a sphere falling in a vertical tube filled with a Poiseuille flow. Then the NSMB software has been extended to be able to take into account multiple moving bodies and the cases of two 2D cylinders falling in free air have been investigated. To prevent the collision of the two cylinders which is not consistent with the chimera method, an elastic shock has been implemented.

Résumé. Ce papier présente les résultats de ces dernières années de recherche à ICUBE, dans l'équipe MECAFLU au sein du groupe "Instabilité, Turbulence, diphasique" sur les simulations des interactions fluide-structure appliquées à la chute des corps en utilisant le solveur NSMB. La première partie de l'étude a été consacrée à l'étude numérique d'une sphère en chute libre dans un tube vertical. Puis le solver NSMB a été étendu pour être en mesure de prendre en compte plusieurs corps en mouvement et les cas de deux cylindres 2D chute libre ont été étudiés. Pour éviter la collision des deux cylindres qui n'est pas possible avec la méthode chimère, un choc élastique a été mis en œuvre.
\end{abstract}

\section{INTRODUCTION}

The interaction of particles on the flow and their trajectories are present in many industrial applications (chemical reactors, transport of particles in the lung airways or blood, transport of fluids containing particles, ...). The characterisation of the behavior of particles can lead to an improvement in these various applications. The problem is simplified as a first step in a single particle in free fall. Many numerical and experimental works have already provided a good insight in the scenario of transition of an unconfined freely moving sphere under the combined action of gravity, buoyancy and hydrodynamic force. Jenny et al $[10,12]$ and Veldhuis et al [28] free motion gives rise to a significantly different scenario as compared to that found in a fixed sphere wake reported by $[8,13,20,27]$. Segre [21] and Segre and Silberberg [22] investigated experimentally a suspension of small neutrally buoyant spheres in a Poiseuille pipe flow and reported an equilibrium radial position at 0.6 radius from the pipe axis. The experimental work of Matas [16] confirmed the results obtained for neutrally buoyant spheres at low Reynolds numbers (based on the pipe diameter) and showed that at higher Reynolds numbers the equilibrium annulus moves toward the wall. Moreover, they reported the existence of a second equilibrium position closer to the pipe axis. For all its practical importance, this configuration is rather difficult

* The authors would like to acknowledge the Direction Informatique (Pôle HPC) of the University of Strasbourg, CINES/GENCI France for supporting this work by providing scientific support and access to computing resources.

${ }^{1}$ ICUBE, Université de Strasbourg / CNRS, 2 Rue Boussingault, 67000 Strasbourg

(C) EDP Sciences, SMAI 2014 
to explore numerically and theoretically. Results of a simulation of a neutrally buoyant sphere at a very low Reynolds number of the pipe flow (0.4) have been published by Yang [31]. They yield an equilibrium position at 0.5 radius. Investigations in two dimensional configurations $[6,32]$ have shown a strong dependence of the equilibrium position on the particle density and predicted an equilibrium position in the symmetry plane of the channel for all sedimenting particles. This is not in contradiction with experimental and numerical threedimensional results because it is well known that sedimenting cylinders behave differently from sedimenting spheres [19].

Takemura and Magnaudet [26] and Takemura et al [25] reported experimental observations of the free ascension of bubbles along a vertical wall. Contaminated bubbles [25] behave much like light rigid spheres and are thus relevant for the mentioned framework. The spheres moving at velocities corresponding to Reynolds numbers ranging from 1 to 100 were observed to migrate away from the wall, witnessing a repulsive lift generated by the wall proximity. This lift was found to decrease with the Reynolds number and the distance from the wall. Zeng [33] investigated the interaction of the sphere wake with a wall numerically. They considered both a non rotating and a freely rotating sphere (like Bagchi and Ballachandar [1] in a shear flow) moving with constant translation velocity parallel to the wall. The investigated Reynolds numbers range from 0.5 to 300 (i.e. extend to a transitional regime characterized by an unsteady wake). They found that the lift reaches a minimum at about $R e=100$ and increases again with increasing Reynolds number. Moreover, they studied the effect of the presence of the wall on the onset of unsteadiness in the wake. At distances $L>d$ ( $L$ being the distance of the center of the sphere from the wall and $d$ the sphere diameter) the unsteadiness sets in at a slightly lower Reynolds number than in an unconfined wake (at $R e=270$ ) but, close to the wall (at $L=0.75 d$ ), its onset is shifted beyond $R e=300$. The free rotation does not change the scenario qualitatively, it only tends to slightly increase the lift coefficient.

This paper begins with a description of the numerical method used within the solver NSMB. The next $\S$ presents the validation of the chimera method for static and moving bodies. $\S 3$ resumes the simulation of the falling of two $2 \mathrm{D}$ cylinders and $\S 4$ deals with the case of a sphere in a Poiseuille flow in a tube.

\section{Numerical SCHEME}

\subsection{Mathematical formulation}

We solve the incompressible Navier-Stokes equations in a cylindrical domain filled with a fluid of uniform density $\rho$ and kinematic viscosity $\nu$ containing a homogeneous moving sphere of diameter $d$ and density $\rho_{s}$ within the NSMB solver [30]. The NSMB solver is a compressible code used, for the present purpose, in the incompressible limit obtained by artificial preconditioning. In agreement with Jenny [12] we non-dimensionalise the velocity by the velocity scale defined by the effective acceleration and the sphere diameter $v_{\text {unit }}=\sqrt{\left(1-\rho_{s} / \rho\right) g d}$ and we express the velocity field with respect to a fixed reference frame. The length scale of non-dimensionalisation is defined by the sphere diameter. Dimensionless quantities based on these velocity and length scales are used throughout the paper. The dimensionless flow equations read as follows:

$$
\begin{gathered}
\frac{\partial \mathbf{v}}{\partial t}+\left[\left(\mathbf{v}-\mathbf{u}_{m e s h}\right) \cdot \nabla \cdot\right] \mathbf{v}=-\nabla p+\frac{1}{G} \nabla^{2} \cdot \mathbf{v} \\
\nabla \cdot \mathbf{v}=0
\end{gathered}
$$

where $\mathbf{u}_{\text {mesh }}$ is the mesh velocity equal to the body translation velocity $\mathbf{u}$ in the body sub-domain and only to its vertical projection in the background one and $G$ is the Galileo number defined by [12] and [28]

$$
G=\frac{\sqrt{\left|\rho_{s} / \rho-1\right| g d^{3}}}{\nu}
$$

with $\rho_{s}$ and $\rho$ the solid and fluid density, $g$ the gravitational acceleration, $d$ the sphere/cylinder diameter and $\nu$ the kinematic viscosity of the fluid. In the case of a falling sphere, the translation and angular velocity ( $\mathbf{u}$ and $\boldsymbol{\Omega})$ obey the motion equations [11]: 


$$
\begin{aligned}
\frac{\rho_{s}}{\rho} \frac{d \mathbf{u}}{d t} & =\frac{6}{\pi} \mathbf{F}_{f l}(\mathbf{v}, p)-\mathbf{i} \\
\frac{\rho_{s}}{\rho} \frac{d \boldsymbol{\Omega}}{d t} & =\frac{60}{\pi} \mathbf{M}_{f l}(\mathbf{v}, p) .
\end{aligned}
$$

where $\mathbf{i}$ is the vertical upward pointing unit vector and $\mathbf{F}_{f l}$ and $\mathbf{M}_{f l}$ are the hydrodynamic force and torque.

\subsection{Chimera method}

Since it is necessary to simulate a non-axisymmetric geometry, Eqs. (1) through (5) are solved by the parallel Navier-Stokes, Multi-Block (NSMB) solver [29] based on a cell-centered finite volume discretisation. The moving geometry has been obtained by implementing the chimera method within the NSMB code. The detailed implementation of the chimera method can be found in the thesis of T. Deloze [4] and its application to a falling cylinder in [5]. We recall here the principles of the chimera method.

The chimera method is based on the management of independent overlapped grids. It significantly simplifies the generation of meshes. It provides a good alternative to unstructured grids. Its principle is to decompose the complex full domain into simple sub-domains independently meshed by curvilinear grids. The only constraint is a superposition of grids to allow the link of the flow description. Beyond simplifying the mesh generation, this technique offers a powerful solution to manage moving bodies.

The pioneers of the chimera method are Benek et al. [2] and the method has subsequently been improved and adapted to many cases. The huge possibilities offered by this approach explain its successful use in many and diverse applications like high-speed reaction flow, blood flow, combustion, aerodynamics, flow around ships, separation of the spacecraft orbiter from boosters or aerodynamic noise. It is associated with other numerical tools to increase efficiency (such as Automatic Mesh Refinement (AMR) coupling with chimera method [9] for example).

The chimera process is detailed for structured grids by Thompson [17]. Here an overview and basic definitions of chimera method is discussed, inspired by the definition of Landmann [14]. The process of chimera method is divided into four main steps :

- $1^{\text {st }}$ step : Detection of overlapped cells. This process consists in finding which cells are overlapped by other cells. The test is based only on the coordinates of the cells.

- $2^{\text {nd }}$ step : Determination of the overlapped cell status. Three kinds of overlapped cells exist [14] :

a) Interpolated cells : the flow data are obtained by interpolation on other overlapping grids.

b) Calculated cells : the values come from the resolution of the equations governing the flow.

c) Hole cells (or blanked/masked cells) : the values of this kind of cells are never used in the discretization schemes or overlapped boundary conditions and they are not interpolated nor calculated.

- $3^{\text {rd }}$ step : Calculation of the interpolations parameters. In this step, the donor cells and the associated weights that compose the interpolation are evaluated for each valid overlapped cell.

- $4^{\text {th }}$ step : Calculation of the interpolation values.

The main advantage of our method over the method proposed by Landmann is in a smart determination of overlapped cell kinds based on the local distance to the wall which enforces the resolution of the Navier-Stokes equation on cells near walls by the grid owing the wall boundary condition and is totally automatic with no user intervention. It allows to manage a configuration with multiple walls (sphere and tube) and automatically adapts to the change in configuration (displacement of the sphere in the tube). 


\begin{tabular}{|c|c|c|c|c|c|c|c|}
\hline Configurations & $\operatorname{Re}$ & $\mathrm{St}$ & Vortex & $C_{d 1}$ & $C_{l 1}$ & $C_{d 2}$ & $C_{l 2}$ \\
\hline 1.5D Present & 200 & 0.168 & No & 0.877 & 0.0204 & -0.195 & 0.0548 \\
\hline 1.5D Borazjani et al. (2009) & 200 & 0.174 & No & 1.1121 & 0.024 & -0.216 & 0.05 \\
\hline 1.5D Meneghini et al. (2001) & 200 & 0.167 & No & 1.06 & 0.03 & -0.18 & 0.06 \\
\hline 1.5D Slaouti et al. (1992) & 200 & 0.14 & No & 0.83 & 0.2 & -0.17 & 0.3 \\
\hline 2.5D Present & 160 & 0.123 & No & 0.826 & 0.0198 & -0.179 & 0.143 \\
\hline 2.5D Mahir et al. (1996) & 160 & 0.1457 & No & unk & unk & unk & unk \\
\hline $5 D$ Present & 160 & 0.174 & Yes & 1.02 & 0.462 & 0.815 & 1.746 \\
\hline 5D Mahir et al. (1996) & 160 & 0.1911 & Yes & unk & unk & unk & unk \\
\hline
\end{tabular}

TABLE 1. Comparison of results for static configuration. Reynolds number, Strouhal number, vortex formation between cylinders, mean drag coefficient $\left(C_{d}\right)$, lift coefficient amplitude $\left(C_{l}\right)$ for two identical stationnary cylinders and a gap between cylinders equals to $1.5 D, 2.5 D$ and $5 D$. "unk" for Unknown.

\section{VAlidation OF THE CHIMERA METHOD}

\subsection{Static Case}

At first, a static case was computed and compared with the literature. The flow around two identical and static circular cylinders is simulated for different gaps between cylinders and two different values of Reynolds numbers : $R e=160$ and $R e=200$. The Strouhal numbers, drag and lift coefficients obtained are compared with the simulations performed by Borazjani et al. [3], Meneghini et al. [18], Slaouti et al. [24] and the experimental results obtained by Mahir et al. [15].The results are summarized on Table 1.

The results obtained exhibit a good overall agreement. As experimentally observed by Mahir et al. [15] Von Kármán vortices form between cylinders when the gap is equal to $5 D$ and $R e=160$ whereas for $R e=200$ no vortex forms between cylinders in configurations $2.5 \mathrm{D}$ and $1.5 \mathrm{D}$. The Strouhal number obtained for configurations $1.5 D$ is in agreement with the existing results, whereas slight differences are obtained in configurations corresponding to a gap of $2.5 D$ and $5 D$ with the experimental results of Mahir et al (2009). The drag and lift coefficients obtained exhibit a good compliance with Borazjani et al. and Meneghini et al. for configuration 1.5D. Drag and lift coefficients for configurations $2.5 \mathrm{D}$ and $5 \mathrm{D}$ can not be compared as they are not mentioned by Mahir et al.

\subsection{Vortex-Induced Vibrations}

We consider an incompressible Newtonian fluid past a system of two identical elastically mounted cylinders that are free to vibrate in transverse direction of the flow (Figure 1a)). The equation of motion of a cylinder is obtained from the Newton's second law of motion:

$$
m \frac{\partial^{2} Z}{\partial t^{2}}+c \frac{\partial Z}{\partial t}+k y=F
$$

where $m$ is the mass of the cylinder, $Z$ the vertical position, $c$ the parameter governing the damping, $k$ the stiffness of the spring and $F$ the force applied to the cylinder by the flow. 


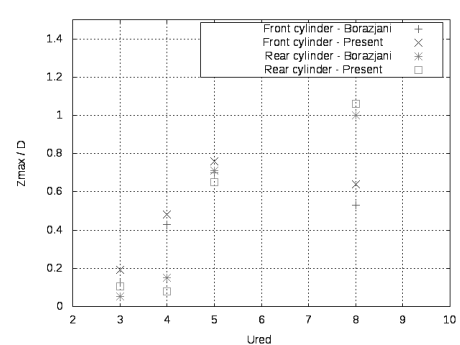

a)

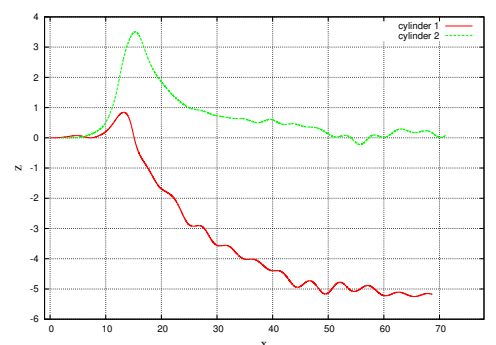

b)

Figure 1. a) Comparison with Borazjani et al. [3] of maximum vibration amplitude vs reduced velocity ( for $U_{\text {red }}=3,4,5$ and 8 ) of two 1 d.o.f cylinders in tandem with a gap of $1.5 D, M_{\text {red }}=2$, $\xi=0$ for $R e=200$; b) Trajectories of the cylinders for Configuration A and $G a=300, \beta=2$

The natural frequency $f$ and critical damping factor $c_{c r}$ of the cylinder are given by

$$
\omega=2 \pi f=\sqrt{k / m}, \quad c_{c r}=2 \sqrt{m k}=2 k \omega .
$$

Eq. 6 can be formulated in a non-dimensional form as [3]:

$$
\frac{\partial^{2} Q}{\partial t^{2}}+\frac{4 \pi \xi}{U_{\text {red }}} \frac{\partial Q}{\partial t}+\frac{4 \pi^{2}}{U_{\text {red }}^{2}} Q=\frac{1}{2 M_{\text {red }}} C
$$

The non-dimensional parameters are defined as: reduced coordinate $Q=Z / D$, non-dimensional damping coefficient $\xi=c / c_{c r}$, reduced velocity $U_{r e d}=U /(f D)$, reduced mass $M_{r e d}=m /\left(\rho D^{2}\right)$, non-dimensional force coefficient $C=F /\left(\frac{1}{2} \rho U^{2} D\right)$.

In our solver, the formulation used is similar than the one used by Shiels et al. [23]:

$$
m^{*} \frac{\partial^{2} Q}{\partial t^{2}}+b^{*} \frac{\partial Q}{\partial t}+k^{*} Q=C
$$

thus

$$
\left(m^{*}, b^{*}, k^{*}\right)=\left(2 M_{r e d}, \frac{8 \pi \xi M_{r e d}}{U_{\text {red }}}, \frac{8 \pi^{2} M_{r e d}}{U_{r e d}^{2}}\right)
$$

The motion of the cylinder is not imposed and must be computed at each time step. To do so, a Newmark algorithm is used to compute the motion of the cylinders at next time step. This algorithm, based on the hypothesis of linear acceleration solves the equations of motion implicitly. We have performed four simulations of the flow past two elastically mounted cylinders in tandem. According to the simulations made by Borazjani et al. [3] the cylinders are placed at $L / D=1.5$ apart in a fluid at $R e=200$ with $M_{\text {red }}=2, \xi=0$ and $U_{\text {red }}$ varying in the range $U_{\text {red }}=3,4,5,8$. The static state computed previously is taken as initial condition of the computation. The oscillations of the cylinders for all 4 configurations are plotted on Figure 2. The maximum amplitude of the vibrations along the $y$ direction when the quasi-steady state is reached is then compared to the results obtained by Borazjani in Figure 1b). An overall good agreement is observed for all our simulations. A discussion about the results of this vortex induced vibrations system can be found in [3]. 

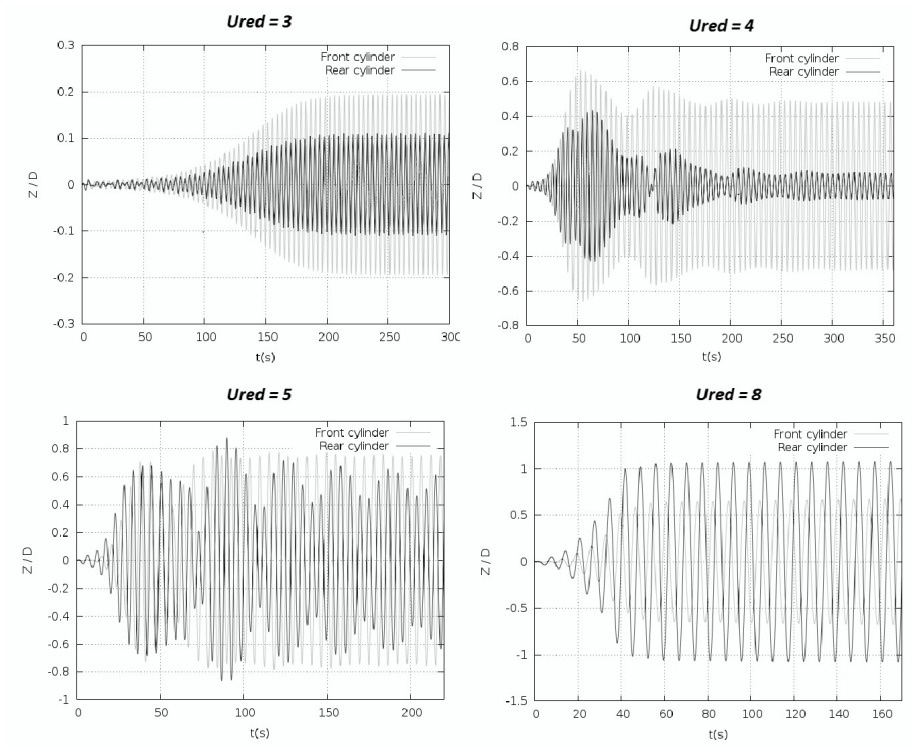

Figure 2. Transverse position of the cylinders versus time for $U_{\text {red }}=3,4,5,8$ of two 1 d.o.f cylinders in tandem with a gap of $1.5 D, M_{r e d}=2, \xi=0$ for $R e=200$.

\section{TWO-Dimensional Simulation OF THE FALL OF FREE CYLINDERS}

\subsection{Numerical method}

The release at zero speed of two identical circular cylindrical particles and their fall under the action of gravity in a fluid at rest is considered. The domain used in the following simulations is to $45 d$ in length by $50 d$ in wide and contains 389847 cells. The domain is large enough to consider that no confinement is induced.

In order to follow the motion, the background Cartesian mesh is attached to a frame that follows the barycenter of the system of cylinders along the $\mathrm{x}$-direction and the $\mathrm{z}$-direction. The polar meshes corresponding to the cylinders are free to move along the $\mathrm{x}$ and $\mathrm{z}$ directions and to rotate.

The only forces acting on the cylinders are the gravity along the x-direction and the resultant forces of the fluid. The equation of motion of a two-dimensional circular particle is obtained from the second Newton's law. Each cylinder has three degrees of freedom. In a non-dimensionalised formulation the particles equations can be written as :

$$
\beta \ddot{x}=g(\beta-1)+\frac{4}{\pi} F_{x}, \beta \ddot{z}=\frac{4}{\pi} F_{y}, \beta \ddot{\alpha}=\frac{8}{\pi} M_{z},
$$

with $F$ being the aerodynamic forces and $M$ the moment. The equations are adimensionalised to obtain a terminal velocity equals to $v_{x, \max }=1$.

The results are classified in three different configurations depending of the initial arrangement of the released cylinders. Configuration $\mathrm{A}$ is defined by a tandem arrangement and a gap of $2 d$ horizontally. Configuration $\mathrm{B}$ is similar to configuration $\mathrm{A}$ but with a gap equal to $5 d$. Configuration $\mathrm{C}$ is characterized by a horizontal gap of $3 d$ and a vertical gap of $1 d$. The density ratio $\beta=\frac{\rho_{s}}{\rho_{f}}$ is set to $\beta=2$ in all the simulations performed while the Galileo number was varied starting from $G a=100$ to $G a=350$.

Simulations have shown that collisions between cylinders can occur. As it is not consistent with the chimera method, it was necessary to implement a collision strategy. For this study a simple elastic collision was chosen. The procedure is the following, at each time step the cylinders positions are extrapolated through time, if the extrapolated positions induce a collision, the velocity of the cylinders are updated using the kinetic energy and 


\begin{tabular}{|c|c|c|} 
Configuration & $\begin{array}{c}\text { Pos. of the } 1^{\text {st }} \text { cylinder } \\
(\mathrm{x}, \mathrm{z})\end{array}$ & $\begin{array}{c}\text { Pos. of the } 2^{\text {nd }} \text { cylinder } \\
(\mathrm{x}, \mathrm{z})\end{array}$ \\
\hline & $(0,0)$ & $(2,0)$ \\
$\mathrm{A}$ & $(0,0)$ & $(5,0)$ \\
$\mathrm{B}$ & $(-1,3)$ & $(0,0)$ \\
C & $(0)$
\end{tabular}

TABLE 2. Summary of the configurations studied

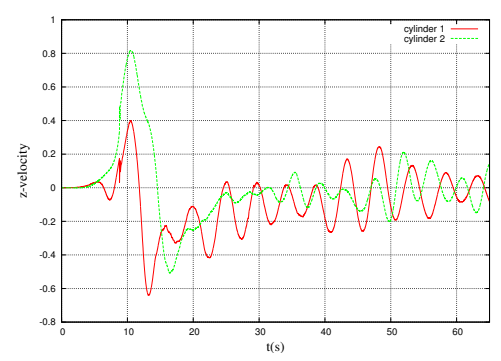

(A) $\mathrm{Z}$ velocity vs time

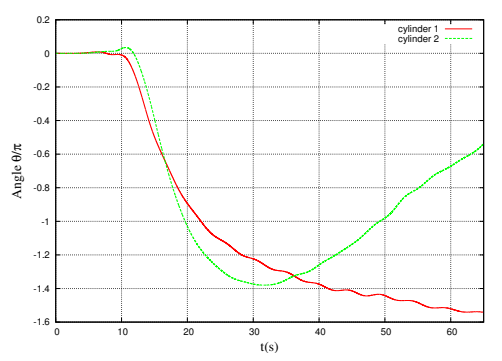

(в) angle vs time

Figure 3. Configuration A and $G a=300, \beta=2$

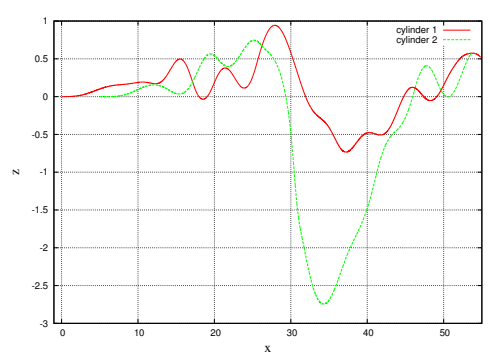

a)

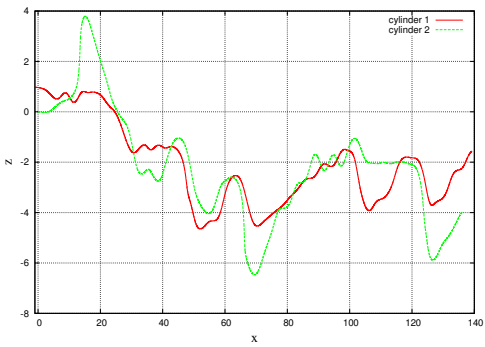

b)

Figure 4. Trajectories of the cylinders for Configuration $\mathrm{B}(\mathrm{a})$ and $\mathrm{C}(\mathrm{b})$ for $G a=300$ and $\beta=2$

momentum conservation relations. In the case of multiple collision occurring at the same time step when more than two spheres/cylinders are simulated, the collision of the closest extrapolated cylinders is treated firstly.

\subsection{Results}

In figure $1 \mathrm{~b}$ ), the trajectories of the cylinders for configuration A and $G a=300$ are plotted. Transverse velocity and angle versus time are plotted in figure 3. A drafting, kissing and tumbling scenario [7] is observed. Cylinder 2 is sucked in the wake of the cylinder 1 with an increasing velocity then it collides and moves around. The cylinder 2 takes the lead and both cylinders separate. When the D.K.T. scenario is complete the cylinders are too far away to interact and act as independent particles with a similar behavior as obtained in [5]. The discontinuity of the velocities observed on figure $3 \mathrm{a}$ at time $t \simeq 9 \mathrm{~s}$ is due to the update of the velocities after the collisions between both cylinders. In Figure 5a and Figure 5b the result of the configuration $\mathrm{B}$ for $G a=300$ is plotted. One can observe that the global behavior is different. Indeed, the cylinders do not separate as observed previously. The trajectories observed exhibits more complexity and higher transverse velocity oscillations are observed. Similarly to the configuration A, the cylinder which is initially downstream catches-up the first one 


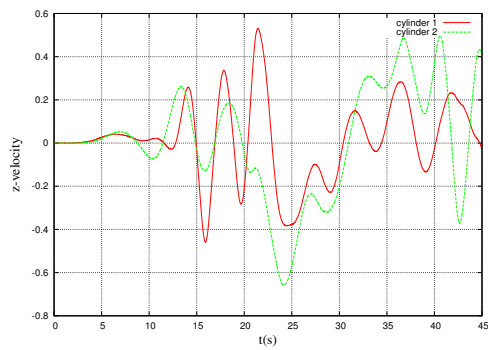

(A) $\mathrm{Z}$ velocity vs time

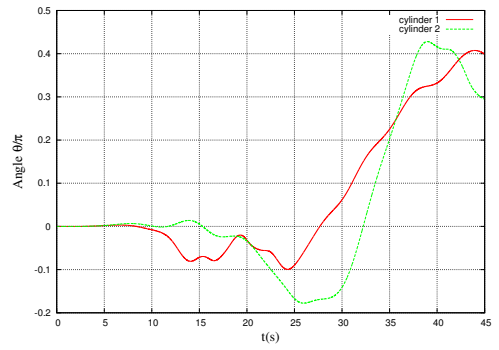

(в) angle vs time

Figure 5. Configuration B and $G a=300, \beta=2$

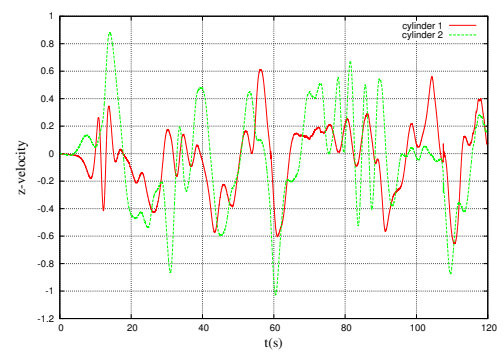

(A) $\mathrm{Z}$ velocity vs time

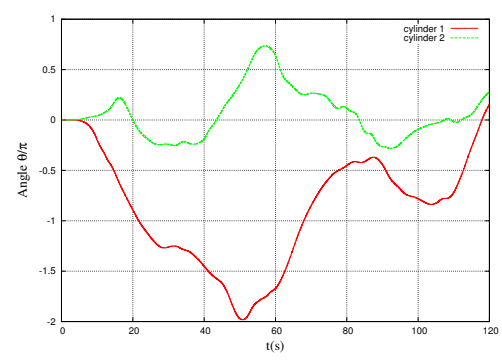

(в) angle vs time

Figure 6. Configuration $\mathrm{C}$ and $G a=300, \beta=2$

and takes the lead. The wake of the cylinder 1 induces larger transverse oscillations than the cylinder 2 .

In figure $4 \mathrm{~b}$ ) and Figure 6, a simulation is performed for configuration $\mathrm{C}$ at $G a=300$. Again as in configuration $\mathrm{B}$, the cylinders have a complex behavior and do not separate. A slight collision occurs at $t \simeq 108 \mathrm{~s}$ as can be seen on figure 6a).

\section{Three-Dimensional simulation of the Flow Around a sphere in a Poiseuille FLOW IN A TUBE}

We solve the incompressible Navier-Stokes equations in a cylindrical domain filled with a fluid of uniform density $\rho$ and kinematic viscosity $\nu$ containing a homogeneous sphere of diameter $d=2$ and density $\rho_{s}$. The sphere is positioned into a Poiseuille flow imposed at the inlet. The tube is simulated by a cylindrical domain of diameter $D=5 d$. The sphere's center is in a horizontal plane situated $15 d$ downstream of the inflow (the bottom cylinder basis) and 40d upstream of the outflow in order to capture the wake behind the sphere. The overall cylinder height is $55 d$. The sphere is embedded into a spherical sub-domain, which will allow us to simulate its motion. The domain is partitioned into 128 blocks and meshed with 2,599,974 finite volume cells. In order to identify the mechanisms of a particle falling freely into a Poiseuille flow we will first study static cases of a particle positioned at different distance from the axis, then we will allow the particle to rotate, and eventually we will let the particle fall freely. Our study cover the Reynolds $R e=(V d) / \nu$ equals to 100,150, 200, 250 and 300. Since the input velocity $V$ is set to 1 and the diameter $d$ of the sphere is fixed to 1, we have a relation linking the Galileo number $G a=\sqrt{\left|\rho_{s} / \rho-1\right| g d^{3}} / \nu$ and the Reynolds number for the free fall case : $G a=\sqrt{g} R e$. In figure 8 a the drag coefficient versus the ratio $d / D$ is plotted. One can observe that an increase of the Reynolds number induces a decrease of the drag coefficient. The drag coefficient is also reduced when the sphere is moved away from the center. When the sphere is placed at the center of the tube the drag is maximum due to a symmetric 


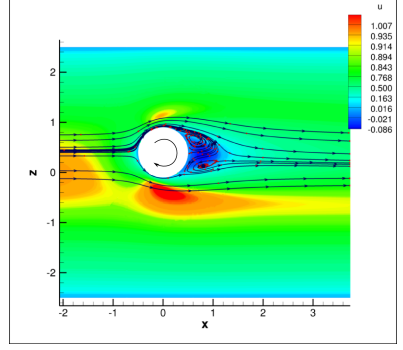

(A) $d / D=0.16$

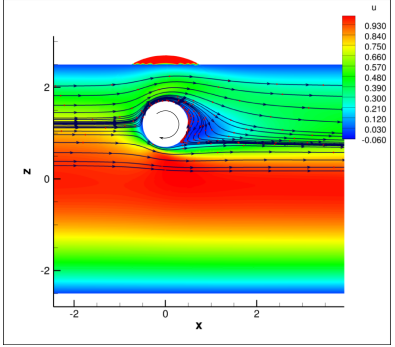

(в) $d / D=0.48$

FiguRE 7. Velocity profile in the plane $y=0$

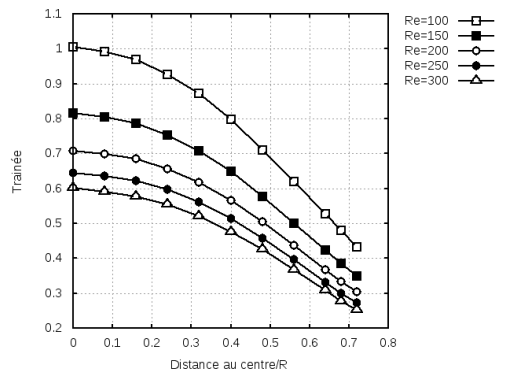

(A) Drag coefficient vs $d / D$

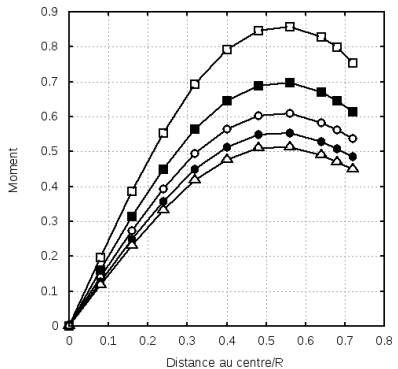

(в) Momentum $z$ vs $d / D$

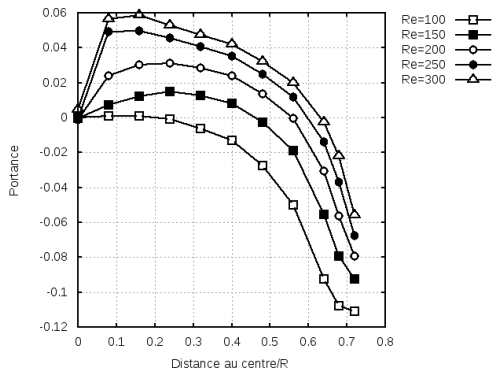

(c) Lift vs $d / D$

FiguRE 8. Evolution of the aerodynamic coefficients

recirculation. This recirculation loses its symmetry and strength, and tends to disappear as the particle gets closer to walls of the tube. This phenomenon can be observed in figures $7 \mathrm{a}$ and $7 \mathrm{~b}$. The figure $8 \mathrm{c}$ represents the lift coefficient versus $d / D$. The lift coefficient globally increases together with the Reynolds number, remains small and stays below 0.12. Three stages describing the evolution of the lift can be observed. First, the lift is positive and increases, then it decreases, and eventually it becomes negative. The lift at the center of the tube is null because of the symmetry of the configuration.

On the figure $8 \mathrm{~b}$ the $y$ momentum is plotted. The $x$ and $z$ momentum are negligible compared to the $y$ one. The momentum $y$ is always positive, decreases with the Reynolds and reaches a maximum around $d / D=0.55$. The figure $7 \mathrm{~b}$ shows the streamlines surrounding the sphere and inducing the positive momentum. The second stage of this study is to allow the rotation of the sphere, to understand the contribution of these degrees of freedom. Unsteady simulations are being performed at the sames positions, taking the initial condition from the converge steady state.

\section{REFERENCES}

[1] P. Bagchi and S. Balachandar. Effect of free rotation on the motion of a solid sphere in linear shear flow at moderate Re. Physics of Fluids, 14:2719, 2002.

[2] J. A. Benek, J. L. Steger, and F.C. Dougherty. A flexible grid embedding technique with application to the euler equations. AIAA Paper, 83-1944, 1983.

[3] I. Borazjani and F. Sotiropoulos. Vortex-induced vibrations of two cylinders in tandem arrangement in the proximity-wake interference region. J. Fluid Mech., 621:321-364, 2009.

[4] T. Deloze. Couplage fluide-solide appliqué à l'étude de mouvement d'une sphère libre dans un tube vertical. PhD thesis, Université de Strasbourg, http://scd-theses.u-strasbg.fr/2203, 2011. 
[5] T. Deloze, Y. Hoarau, and J. Dušek. Chimera method applied to the simulation of a freely falling cylinder in a channel. European Journal of Computational Mechanics, 19(5-7):575-590, 2010.

[6] J. Feng, H.H. Hu, and D.D. Joseph. Direct simulation of initial value problems for the motion of solid bodies in a Newtonian fluid Part 1. Sedimentation. Journal of Fluid Mechanics, 261:95-134, 1994.

[7] A.F. Fortes, D.D. Joseph, and T.S. Lundgren. Nonlinear mechanics of fluidization of beds of spherical particles. J. Fluids Mech., 177:407-483, 1987.

[8] B. Ghidersa and J. Dušek. Breaking of axisymetry and onset of unsteadiness in the wake of a sphere. J. Fluid Mech., 423:33-69, 2000.

[9] W.D. Henshaw and D.W. Schwendeman. Parallel computation of three-dimensional flows using overlapping grids with adaptive mesh refinement. Journal of Computational Physics, 227:7469-7502, 2008.

[10] M. Jenny, G. Bouchet, and J. Dušek. Nonvertical ascension or fall of a free sphere in a Newtonian fluid. Physics of Fluids, 15:L9, 2003.

[11] M. Jenny and J. Dušek. Efficient numerical method for the direct numerical simulation of the flow past a single light moving spherical body in transitional regimes. Journal of Computational Physics, 194:215-232, 2004.

[12] M. Jenny, J. Dušek, and G. Bouchet. Instabilities and transition of a sphere falling or ascending freely in a Newtonian fluid. J. Fluid Mech., 508:201-239, 2004.

[13] T.A. Johnson and V.C. Patel. Flow past a sphere up to a Reynolds number of 300. J. Fluid Mech., 378:19-70, 1999.

[14] B. Landmann and M. Montagnac. A highly automated parallel Chimera method for overset grids based on the implicit hole cutting technique. International Journal for Numerical Methods in Fluids, 2010.

[15] N. Mahir and D. Rockwell. Vortex formation from a forced system of two cylinders : Part 1. J. Fluids Struct., 10(5):473-489, 1992.

[16] J.P. Matas, J.F. Morris, and E. Guazzelli. Inertial migration of rigid spherical particles in Poiseuille flow. Journal of Fluid Mechanics, 515:171-195, 2004.

[17] R. L. Meakin. Chapter 11 : Composite overset structured grids, in : Handbook of grid generation, J.F. Thompson, B.K. Soni and N.P. Weatherill. CRC, 1999.

[18] J. Meneghini, F. Saltara, C. L. R. Siqueira, and J. J. A. Ferrari. Numerical simulation of flow interference between two circular cylinders in tandem and side-by-side arrangements. J. Fluids Struct., 15(2):327-350, 2001.

[19] K. Namkoong, J.Y. Yoo, and H.G. Choi. Numerical analysis of two-dimensional motion of a freely falling circular cylinder in an infinite fluid. Journal of Fluid Mechanics, 604:33-53, 2008.

[20] D. Ormières and M. Provansal. Transition to turbulence in the wake of a sphere. Phys. Rev. Lett., 83:80-83, 1999.

[21] G. Segré. Radial particle displacements in Poiseuille flow of suspensions. Nature, 189:209-210, 1961.

[22] G. Segré and A. Silberberg. Behaviour of macroscopic rigid spheres in Poiseuille flow Part 1. Determination of local concentration by statistical analysis of particle passages through crossed light beams. Journal of fluid mechanics, 14(01):115-135, 1962.

[23] D. Shiels, A. Leonard, and A. Roshko. Flow-induced vibration of a circular cylinder at limiting structural parameters. Journal of Fluid and Structures, 15:3-21, 2000.

[24] A. Slaouti and P. Stansby. Flow around two circular cylinders by the random-vortex method. J. Fluids Struct., 6(6):641-670, 1992.

[25] F. Takemura and J. Magnaudet. The transverse force on clean and contaminated bubbles rising near a vertical wall at moderate Reynolds number. J. Fluid Mech., 495:235-253, 2003.

[26] F. Takemura, S. Takagi, J. Magnaudet, and Y. Matsumoto. Drag and lift forces on a bubble rising near a vertical wall in a viscous liquid. J. Fluid Mech., 461:277-300, 2002.

[27] A.G. Tomboulides and S.A. Orszag. Numerical investigation of transitional and weak turbulent flow past a sphere. Journal of Fluid Mechanics, 416:45-73, 2000.

[28] C.H.J. Veldhuis and A. Biesheuvel. An experimental study of the regimes of motion of spheres falling or ascending freely in a Newtonian fluid. International Journal of Multiphase Flow, 33(10):1074-1087, 2007.

[29] J. Vos, A. Rizzi, D. Darracq, and E. Hirschel. Navier-Stokes solvers in European aircraft design. Progress in Aerospace Sciences, 38:601-697, 2002.

[30] J. Vos, A. Rizzi, A. Orjon, E. Chaput, and E. Soinne. Recent Advances in aerodynamics inside the NSMB (Navier-Stokes Multiblock) Consortium. AIAA paper, 98-0225, 1998.

[31] B.H. Yang, J. Wang, D.D. Joseph, H.H. Hu, T.W. Pan, and R. Glowinski. Migration of a sphere in tube flow. Journal of Fluid Mechanics, 540:109-131, 2005.

[32] Z. Yu, N. Phan-Thien, Y. Fan, and R.I. Tanner. Viscoelastic mobility problem of a system of particles. Journal of NonNewtonian Fluid Mechanics, 104(2-3):87-124, 2002.

[33] L. Zeng, S. Balachandar, and P. Fischer. Wall-induced forces on a rigid sphere at finite Reynolds number. J. Fluid Mech., 536:1-25, 2005. 